\title{
Filigrane
}

Écoutes psychanalytiques

\section{Le double cadre : le processus de la consultation au CCTP Jean-Favreau}

\section{Danielle Kaswin}

Volume 27, numéro 2, 2018

La consultation psychanalytique aujourd'hui, entre héritages et remaniements

URI : https://id.erudit.org/iderudit/1055748ar

DOI : https://doi.org/10.7202/1055748ar

Aller au sommaire du numéro

Éditeur(s)

Revue Santé mentale au Québec

ISSN

1192-1412 (imprimé)

1911-4656 (numérique)

Découvrir la revue

Citer cet article

Kaswin, D. (2018). Le double cadre : le processus de la consultation au CCTP Jean-Favreau. Filigrane, 27(2), 39-53. https://doi.org/10.7202/1055748ar
Résumé de l'article

La consultation psychanalytique a pris toute sa place dans la clinique et la transmission psychanalytiques, à côté du soin, avec l'ouverture des cliniques gratuites comme le souhaitait Freud. Le Centre de consultations et de traitements psychanalytiques Jean-Favreau s'est inscrit dans cette continuité. Une recherche au sein d'un groupe de pairs se poursuit depuis de nombreuses années selon les modalités d'un travail associatif groupal. Les échanges inter-analytiques à partir de séquences cliniques s'appuient sur l'écoute en second, à partir de l'institutionnalisation de la dualité consultation/traitement, et permettent l'évaluation de la pertinence des indications. La tiercéisation du dispositif, soit « le double cadre », donne son titre à l'article. 


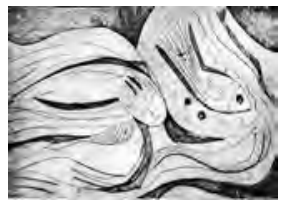

\title{
Le double cadre: le processus de la consultation au CCTP Jean-Favreau
}

\author{
Danielle Kaswin
}

\begin{abstract}
Résumé: La consultation psychanalytique a pris toute sa place dans la clinique et la transmission psychanalytiques, à côté du soin, avec l'ouverture des cliniques gratuites comme le souhaitait Freud. Le Centre de consultations et de traitements psychanalytiques Jean-Favreau s'est inscrit dans cette continuité. Une recherche au sein d'un groupe de pairs se poursuit depuis de nombreuses années selon les modalités d'un travail associatif groupal. Les échanges inter-analytiques à partir de séquences cliniques s'appuient sur l'écoute en second, à partir de l'institutionnalisation de la dualité consultation/traitement, et permettent l'évaluation de la pertinence des indications. La tiercéisation du dispositif, soit «le double cadre», donne son titre à l'article.
\end{abstract}

Mots clés: consultation; écoute interanalytique; tiers; recherche en psychanalyse; cadre analytique

\begin{abstract}
The psychoanalytic consultation has taken its full place in psychoanalytic clinic and transmission, alongside care, with the opening of free clinics as Freud wished. The Center for Psychoanalytic Consultations and Treatments Jean-Favreau is part of this continuity. Research within a peer group has been going on for many years in this center, according to the modalities of a group associative work. Interanalytic discussions, based on "secondary listening" of clinical sequences, draw on the institutionalization of the consultation / treatment duality. This allows the evaluation of the relevance of therapeutic indications. The third party setting of the setting, called «the double frame», gives its title to this paper.
\end{abstract}

Keywords: consultation; interanalytical listening; third party; research in psychoanalysis; analytical framework

— reud, au sujet des hypothèses que la psychanalyse met au travail, précise: «il est difficile de dire s'il faudrait les considérer comme des postulats ou des produits de nos recherches» (Freud, 1938).

Toute la problématique de la recherche en psychanalyse se trouve condensée dans cette phrase. La question de la dimension scientifique de la psychanalyse et de la recherche en psychanalyse reste en effet polémique. 
Elle ne peut répondre aux exigences de critères permettant d'évaluer l'efficacité thérapeutique de la psychanalyse à partir d'indicateurs de mesures positivistes, ou encore de critères cliniques nosographiques ou sociologiques. La spécificité de son épistémologie propre impose une approche intrinsèque à la psychanalyse et aux modalités du fonctionnement psychique dans sa prise en compte de l'inconscient. Un travail toujours à remettre en question à travers l'écart théorico-clinique auquel les analystes sont toujours confrontés dans leur pratique.

Toute réflexion sur la consultation ou le premier entretien, quel qu'en soit le site (Donnet, 2005), ne peut se concevoir qu'en articulation étroite avec une réflexion sur la situation analytique et la problématique de l'éthique psychanalytique, ainsi qu'avec un questionnement sur la filiation et la transmission.

Il n'est plus nécessaire de rappeler l'importance des premières rencontres pour chaque patient, pour chaque traitement psychanalytique, pour chaque analyste et la place qu'occupe le processus de la consultation dans la transmission et l'enseignement de la psychanalyse, que ce soit en pratique privée ou dans un centre de consultations ou de soins. Au CCTP JeanFavreau, la rencontre implique généralement au minimum deux entretiens, parfois davantage, et elle peut nécessiter une répétition des entretiens, qui s'effectuent alors à un rythme espacé. La distinction entre consultation et traitement ne constitue donc pas toujours une limite infranchissable. Ces consultations peuvent prendre une dimension de traitement d'essai, préconisé par Freud, mais abandonné aujourd'hui ${ }^{1}$. Il arrive que les "consultations» qui se répètent à un rythme espacé deviennent des consultations dites «thérapeutiques».

La rencontre avec un nouveau patient revivifie pour l'analyste sa première rencontre avec un analyste et son propre engagement dans l'analyse, ses émotions, ses angoisses, ses attentes. C. Stein (2011) l'a appelée la double rencontre: l'analyste contacte en lui, lors de chaque premier entretien avec un nouveau patient, le demandeur qu'il a été.

L'appropriation de la fonction analytique s'inscrit dans un parcours complexe qui, à partir de l'analyse personnelle, demande un long processus de maturation, à travers la formation, l'enseignement, la reconnaissance par ses pairs. Le niveau secondarisé d'un savoir transmis est antinomique avec le processus psychanalytique. «On n'apprend pas la technique psychanalytique dans les livres", a soutenu Freud en substance à propos de l'analyse sauvage (Freud, 1910, p. 41). Le psychanalyste est confronté à l'inconnu de 
soi et de l'autre; sa position n'est jamais acquise et, toujours sollicitée dans la dynamique de la rencontre, elle doit sans cesse se dégager du colloque singulier de l'intimité de la séance pour inscrire le tiers en position symbolique, et ainsi permettre que l'objet du transfert laisse la place à l'investissement de la fonction analytique.

«Il est difficile de pratiquer la psychanalyse en solitaire. C'est une entreprise sociale par excellence», écrit Freud à Groddeck, le 21 décembre 1924 (Bolzinger, 2012, p. 203; Groddeck, 1977). Les analystes sont inscrits dans un champ institutionnel où fonctionnent des alliances, des problématiques transférentielles résiduelles inévitables, des identifications intergénérationnelles, où s'expriment des idéaux, des répétitions, voire des interdits de pensée. Inévitablement, des oublis, des dénis, des clivages accompagnent certains fonctionnements narcissiques, transgressifs, parfois pervers. Mais n'est-ce pas l'essence même du travail analytique que de permettre le déploiement du fonctionnement psychique, de valoriser la puissance de la pensée face aux débordements du pulsionnel et face à l'emprise de la répétition? In fine, le but de l'analyse est aussi de permettre le transfert du transfert analytique vers d'autres objets. La consultation psychanalytique a pour fonction d'évaluer cette potentialité à travers le processus qui peut s'y déployer.

Les recherches théorico-cliniques se trouvent à la croisée des parcours individuels, des impasses cliniques et des crises institutionnelles, et le cheminement métapsychologique freudien en est l'exemple princeps. Nous pourrons en saisir l'impact à travers un bref rappel de l'histoire des cliniques gratuites.

\section{Les institutions et le travail de consultation}

Dans un monde social se trouvant en une crise sans précédent, confrontés aux problèmes de pauvreté, d'inégalités sociales, de migrations, de carences, de violences, de racisme et de xénophobie, les centres qui proposent une approche psychanalytique et des consultations ont plus que jamais leur place et se trouvent également devant la nécessité de sauvegarder la psychanalyse.

Ce sont les problématiques sociales de cet ordre qui ont conduit les analystes du courant social-démocrate proches de Freud à s'investir dans le mouvement des cliniques gratuites.

L'importance de ce mouvement et son impact dans le développement de la psychanalyse viennent de la place prépondérante qu'il a occupée dans 
la formation et la transmission de la pratique, de la clinique et de la théorie psychanalytique. Les aléas politiques et financiers vont complexifier l'ouverture de ces établissements, que ce soit à Berlin, Vienne ou Budapest où les analystes ne ménagent pas leurs efforts avec pour objectif d'augmenter le nombre des analystes, et donc de créer des lieux de formation dans un but de recherche et de faire bénéficier un plus grand nombre de personnes d'un traitement psychanalytique. Avec le développement d'instituts au sein des cliniques, la formation se dote de règles qui introduisent, à côté de l'analyse «didactique», des supervisions de cures et des séminaires d'enseignement théorique. L'Institut de Berlin est le lieu où s'élabore la standardisation de la formation à l'API avec la création par Eitingon en 1925 de la Commission internationale d'enseignement théorique.

Le CCTP Jean-Favreau s'inscrit dans la lignée des cliniques gratuites. Dans ses débuts, le CCTP fonctionne sur le mode du dispensaire privé avec la collaboration et le soutien financier de Marie Bonaparte. Il est inauguré en 1954 en même temps que l'Institut de formation dont il dépend. L'Institut est dirigé par S. Nacht qui a passé quelques mois à Vienne et a fréquenté l'Ambulatorium. Les divergences sur les critères de sélection et de formation entre S. Nacht et J. Lacan viennent alors d'aboutir à la scission de 1953, avec la création de la Société Française de Psychanalyse (SFP).

Dès son ouverture donc, la mission du CCTP Jean-Favreau est d'une part de permettre de lier l'expérience clinique, l'enseignement et la théorie psychanalytique et, d'autre part, de répondre à une demande sociale émanant de patients pécuniairement démunis ou adressés au Centre par des services hospitaliers.

Les membres dits titulaires (formateurs) de la Société psychanalytique de Paris (SPP) interviennent bénévolement. Ils posent en consultation les indications, et les analystes en formation conduisent tout aussi bénévolement les cures analytiques pour lesquelles ils bénéficient d'une supervision gratuite. La consultation joue un rôle fondamental d'articulation avec les institutions médicales et psychiatriques, tant au niveau de la clinique que de la formation psychanalytique.

Très vite' en 1948, en lien avec le mouvement de la psychiatrie de secteur soutenu par R. Diatkine et S. Lebovici, les tutelles administratives sanitaires françaises reconnaissent le CCTP comme une institution de soins. Par cette convention et le financement qui en découle, la vocation strictement psychanalytique du CCTP est reconnue dans une visée socio-thérapeutique. Les consultants et les analystes traitants sont rémunérés. Ce financement 
public différencie le Centre de ceux qui l'ont historiquement précédé. Ce ne sont plus seulement les titulaires qui vont effectuer le travail de consultation, ni seulement les analystes en formation qui assureront les analyses. De plus, pour répondre à la nécessité et l'exigence de soins, le panel des traitements se diversifie: cure classique, face-à-face, psychodrame, traitement de groupe.

Les trois axes que représentent les traitements, la formation et la recherche ont toujours structuré le fonctionnement du Centre avec le maintien d'une activité centrée sur les cures analytiques classiques.

Pendant longtemps, les médecins directeurs se sont appliqués à limiter l'incidence institutionnelle et ont privilégié la responsabilité individuelle et la confidentialité. Ils ont orienté leurs recherches sur la gratuité et sur l'extension et la diversification des modalités thérapeutiques associées aux problématiques d'indications et de contre-indications.

Lorsque J.-L. Donnet en 1989 prend la direction du CCTP, il initie un travail de recherche au long court sur les modalités consultatives autour de la dualité psychanalyste consultant/psychanalyste traitant et le double cadre. La consultation est menée par un analyste, mais c'est toujours un autre analyste qui prend en charge le traitement. Cette spécificité appelée «la disjonction consultation-traitement » implique que l'analyste consultant, soit celui rencontré lors du premier rendez-vous, n'est jamais l'analyste traitant. Un patient qui suit un traitement au Centre rencontre toujours deux analystes. Les patients sont informés dès le début qu'ils n'engageront pas de traitement avec l'analyste consultant. Cette modalité de fonctionnement à laquelle nous avons donné le nom de «double cadre» inscrit la référence à un fonctionnement institutionnel et introduit d'emblée une référence tierce, autrement dit une différenciation sujet et fonction, c'est-à-dire la reconnaissance de la différence entre la personne de l'analyste et sa qualité d'analyste, et inscrit toute prise en charge dans un référentiel collectif et culturel. Cette différenciation permet la distinction d'un transfert à double vertex: «le transfert pour interpréter» qui renvoie à la réalité d'une situation primitive et concerne l'implication de l'analyste en tant que personne réelle, et «le transfert à interpréter» qui renvoie à l'analyste en fonction (Donnet, 1995).

\section{La consultation au CCTP}

En préambule, précisons que toute rencontre analytique contient une dimension consultative; elle n'est pas obligatoirement psychanalytique d'emblée, même si elle est menée par un psychanalyste. Elle va ou non le devenir en fonction de ce qui se passe dans la rencontre, et en après-coup. 
L'impact des premières rencontres relève pour une part des effets d'un surinvestissement spécifique du monde intérieur, qui provoque un vacillement des repères habituels et ouvre le champ de la réalité psychique. L'évaluation se fera à l'aune des transformations perceptibles durant l'entretien, qui peut alors prendre éventuellement une dimension de traitement d'essai. L'intelligibilité du processus de la rencontre apparaît chez le patient lorsqu'il perçoit ce qu'est l'association libre, ce qui laisse présager d'une capacité transférentielle qui pourra glisser du transfert sur l'objet (la personne de l'analyste) au transfert sur la méthode (le fonctionnement psychique, ainsi que l'a théorisé J.-L. Donnet, 1995).

Ce qui caractérise le CCTP est que l'institution, avec la notion de «double cadre», fonctionne comme tiers, et qu'en elle-même elle a une valeur symbolisante. C'est-à-dire qu'il s'agit d'une notion spécifique structurale: avec cette "dissociation consultation-traitement», un "cadre du cadre» est introduit dès la première consultation, quelle que soit l'indication à venir. La dialectique transféro-contre-transférentielle s'organise par rapport à cet élément fondamental.

Cette référence tierce, c'est aussi ce que recherchent un certain nombre de patients lorsqu'ils viennent demander les coordonnées d'un analyste ou lorsqu'ils se trouvent en difficulté dans leur démarche analytique.

Cela implique un nécessaire questionnement sur l'interruption du processus transférentiel qui a pu s'installer avec l'analyste consultant, avec comme corollaire une évaluation de la capacité de transformation de ce transfert émergent et de ses possibilités de déplacement.

Ce dispositif interroge également la position de l'analyste, sa conception de la théorie psychanalytique et la dimension contre-transférentielle qui découle de cette situation.

$\mathrm{Au}$ CCTP, nous entendons la consultation psychanalytique comme l'exploration d'une potentialité processuelle qui va permettre d'évaluer les aptitudes à la symbolisation et à l'investissement de la méthode psychanalytique à travers l'éventualité d'un transfert sur la parole. Cette consultation diffère donc de ce qu'il est classique de nommer « entretien préliminaire »; la dimension de cooptation réciproque subjective telle qu'elle se produit dans le contexte d'une pratique libérale va se trouver remplacée par une interruption du processus transférentiel.

Les particularités de ce dispositif ont fait l'objet de différents écrits et constituent un travail de réflexion du groupe dit «restreint» qui est présenté plus loin. 
Au-delà des inconvénients d'un travail psychique de séparation et de deuil implicite pour les deux partenaires du couple consultatif, la fonction de consultation et la dualité «analyste consultant/analyste traitant» acquiert une fonction symbolique. À côté de son positionnement personnel d'analyste, le consultant s'inscrit dans une perspective institutionnelle au nom d'une compétence instituée de la psychanalyse.

$\mathrm{La}$ «dissociation consultation/traitement» mobilise la dynamique transférentielle. Le transfert, dans le cadre d'un travail psychique de transformation, correspond au déplacement d'affect d'une représentation sur une autre. La rencontre explore la possibilité d'un devenir analytique. Elle met en jeu et favorise une succession de phénomènes où vont se manifester des répétitions à saisir comme animées par le transfert. L'analyste offre également, dans ce type de consultation, un silence d'écoute. Le patient va, en fonction de ses défenses, investir cette situation d'écoute. L'angoisse qu'elle génère en lui influence sa façon de présenter sa demande et son histoire. Il va dans les bons cas pouvoir découvrir ce qu'est l'association libre et développer un intérêt pour sa vie psychique. Bien évidemment l'exercice n'est pas toujours facile. Cette compréhension, comme cette ouverture, peuvent ne pas être évidentes pour le patient. L'analyste se doit d'explorer avec souplesse les capacités de celui-ci à s'engager dans la rencontre, et s'y ajuster tout en maintenant à la fois une certaine fluidité et la rigueur de la méthode. Il y a quelque chose d'aporétique dans cette simulation de situation analytique car le cadre analytique n'est pas instauré, et il n'y a ni engagement ni temporalité explicités. Les interventions de l'analyste consultant visent à faciliter et à permettre la compréhension de l'association libre. Il tente de repérer les éléments d'actualisation transférentielle avec ou sans intervention interprétative, tel que nous le rapporte Freud.

Dans «Construction dans l'analyse», Freud (1998, p. 276) présente le mouvement psychique d'un ami médecin venu le consulter avec sa femme pour des difficultés sexuelles du couple. Alors que Freud explicite à la jeune femme les conséquences fâcheuses d'une abstinence sexuelle, le mari s'exclame: «L'anglais chez qui vous aviez posé le diagnostic de tumeur cérébrale est bien mort, lui aussi.» Freud insiste sur l'absurdité apparente du propos. Puis il interprète pour le lecteur l'énigmatique «aussi» mis dans le texte en italique. Il s'interroge d'abord: il n'avait pas été question de mort. Il pense que l'homme voulait avec ce mot soutenir son propos et donne sa compréhension de l'intervention de son ami: avec ce patient le diagnostic s'était avéré pertinent, «aussi». Sa femme doit donc écouter ce que lui dit le professeur! 
C'est, écrit Freud, un exemple parfait des confirmations indirectes par associations telles qu'elles surgissent en analyse. Il poursuit en évoquant sans insister les éventuelles pensées inconscientes que peuvent révéler les paroles du mari!

Comme dans toute consultation, lorsque le patient lui-même reste silencieux, ou demande qu'on lui pose des questions, l'analyste est attentif à la manière qu'a le patient d'investir la situation de consultation. Il peut rester silencieux, il accompagne alors le patient dans le silence.

L'analyste peut également intervenir: interroger ce besoin du patient de répondre à des questions, ou poser des questions pour aider celui-ci à prendre la parole. Il lui est possible aussi d'introduire une dimension ludique ou d'intervenir sur un mode psychodramatique, ce qui toutefois pose la question de la séduction, question qui affleure dans toute rencontre, tant du côté du patient que du côté de l'analyste. L'analyste consultant peut aussi proposer une interprétation afin d'en voir les effets.

\section{Vignette clinique: Elle}

L'un d'entre nous, analyste formateur, reçoit en consultation une jeune femme qui a des difficultés à parler et demande que l'analyste lui pose des questions. Après un moment de silence, il intervient: «On n'a pas dû vous écouter souvent lorsque vous étiez enfant.» La patiente demeure silencieuse pendant un certain temps, mais son silence semble d'une autre tonalité, puis elle commence à parler de manière associative.

L'indication posée à la suite d'un second entretien est une cure analytique sur le divan à trois séances par semaine.

Plusieurs années plus tard, l'un d'entre nous découvre en feuilletant le magazine Elle un article sur les lieux parisiens intéressants et économiques, du genre «Paris pas cher». Il voit qu'il y est fait mention du CCTP et de la gratuité des traitements. Une journaliste y interviewe une femme qui raconte son parcours personnel. Elle parle de son analyse et donne des précisions sur sa rencontre avec l'analyse. Elle évoque la consultation au cours de laquelle l'analyste lui avait fait cette intervention: "On n'a pas dû vous écouter souvent lorsque vous étiez enfant", et elle situe à ce moment précis le début de son travail analytique avec sa difficulté à parler, son silence et l'écoute de ce silence.

On peut comprendre à travers ce témoignage que la patiente a pu s'approprier la construction interprétative de l'analyste, accueillir la désimplication 
subjective de l'interprétation pour investir l'espace transitionnel de la situation analytique tout en participant à sa création.

Ce temps initial de consultation et de découverte de la relation et de la méthode analytiques n'est pas cliniquement très différent en pratique institutionnelle et privée. Ce temps consultatif est dominé par la mobilisation pulsionnelle qui permet l'exacerbation des défenses, souvent projectives, tandis que surgissent, dans le cours du discours du patient, des représentations signifiantes. La différence toutefois est bien dans la problématique du double cadre. Il s'avère d'ailleurs que le patient efface cette donnée durant l'entretien; elle réapparaît à la fin de la rencontre. Refoulement? Déni ? «Ah, ce n'est pas avec vous que je poursuivrai?» La difficulté essentielle de s'adresser à quelqu'un avec lequel le patient ne poursuivra pas sa démarche peut cependant, a contrario, ouvrir la voie à une liberté d'investissement de la situation et à une liberté de parole.

\section{Vignette clinique: Myriam}

La patiente, que j'appellerai Myriam, est une jeune femme d'une trentaine d'année, musicienne et chanteuse. Elle est passée, à l'adolescence, du monde agité et trouble des rues de banlieue à l'atmosphère feutrée et rigide d'un conservatoire parisien. Il lui a fallu beaucoup d'efforts d'adaptation et beaucoup travailler dans un univers où elle est décalée, et dont elle ne connaît pas les codes.

Elle consulte pour une grande détresse qui survient à la suite d'une rupture amoureuse. Elle dit: "C'est la première fois que je sortais avec un Noir... » Je suis surprise, je ne relève pas. Il est aussi un musicien. Il l'avait prévenue qu'il était un «électron libre», mais il lui disait des mots d'amour et était plein d'attention, elle y a cru. Elle est anéantie. Elle me précise qu'elle n'a jusqu'alors jamais imaginé qu'elle pourrait faire une telle demande d'aide, que c'est tellement éloigné de son milieu et de sa culture. Nous revenons sur son amoureux, et elle précise que «sortir avec un Noir, c'est une trop grande proximité, ça serait comme sortir avec un cousin, comme sortir avec son père», lui-même musicien, déjanté, «un type formidable qu'elle est vraiment contente d'avoir rencontré». Dois-je préciser que Myriam a la peau très noire?

Quelque peu histrionne, elle pleure, rit, s'énerve: "Vous devez me prendre pour une folle!» s'exclame-t-elle. Je souligne le verbe qu'elle utilise «vous devez» et lui dis: «je dois?» Elle s'interrompt, elle reste en suspens, pensive, et garde le silence. La reprise du verbe en souligne la polysémie, 
l'injonction projective inconsciente d'une imago qui ne laisse pas le choix d'occuper... cette place de folle? Bien évidemment, il y a beaucoup d'élaboration possible autour de la richesse du matériel; on peut questionner la porosité de la censure autour d'un désir œedipien au refoulement fragile, mais ce que je voudrais souligner, c'est la tonalité associative qui s'inscrit dans son discours lorsqu'elle reprend la parole. En effet, elle évoque alors sa grand-mère maternelle qui l'a élevée, les conflits familiaux, et les injonctions séductrices imagoïques qui l'assignaient à une place particulière dont je comprends qu'il s'agissait d'être la mère de la grand-mère, et d'être folle.

L'interprétation dans le transfert dans le hic et nunc de la consultation se situe au niveau manifeste de l'adresse à l'analyste, mais le matériel est riche d'un contenu latent en prise avec l'histoire infantile de Myriam. Il n'est pas question d'explorer le contenu de la fantasmatique psychique, mais d'évaluer ses défenses et sa mobilité psychique.

Beaucoup d'éléments dans la consultation vont dans le sens de la mobilité psychique.

Il s'agit d'une première consultation qui permet de penser à partir des potentialités réflexives et associatives qu'un travail analytique est envisageable et pourra être proposé à la patiente.

Rappelons que, très souvent, le contenu d'une consultation peut se révéler très différent à la consultation suivante. Tel patient pris dans la répétition lors de la première consultation va dérouler un processus associatif lors du second rendez-vous, ou inversement la richesse associative va laisser la place à une pauvreté idéique. La compréhension de ce qui s'est joué entre les deux consultations, comme le surgissement de nouvelles idées, l'émergence de souvenirs et de rêves, permet d'apprécier la mobilité de la vie psychique.

Les patients viennent d'horizons divers, tantôt ils consultent de leur propre chef au CCTP, tantôt ils sont adressés par des centres thérapeutiques ou des services hospitaliers. Ils arrivent avec une quête dont il s'agira de savoir si elle peut se transformer en désir réel d'une cure classique ou d'une psychothérapie. D’autres fois, il s'agit d'une demande de soins qu'on rencontre plus fréquemment en institution hospitalière.

\section{Le temps de l'indication}

Après le temps de l'exploration vient le moment de l'évaluation et de l'indication. L'objectif de la rencontre est d'aboutir à la proposition d'une instauration ou d'une non-instauration de traitement dans un cadre défini. 
Les enjeux de fin de rencontre sont différents en pratique institutionnelle et en pratique libérale, tant du côté de l'indication que du refus.

Dans la consultation au CCTP, l'analyste étant exclu de l'engagement thérapeutique ultérieur, les conditions de faisabilité ne se posent pas dans les mêmes termes. Les changements de registre de l'échange psychanalytique à celui de la réalité de la proposition d'un cadre de travail, ou encore de la problématique du refus, relèvent du travail analytique de consultation. L'analyste consultant apprécie quelle utilisation de la situation analytique le patient va pouvoir faire, son investissement de la fonction portée par l'analyste et du tiers représenté par le cadre ainsi que les caractéristiques du transfert, son intensité. Le transfert sur l'objet analyste est-il mobilisable et potentiellement transférable sur la parole et sur la fonction analysante? L'analyste s'appuie sur la découverte par le patient du site analytique à travers la mobilisation de ses défenses au cours de la rencontre. Quelles sont les possibilités d'appropriation subjective de la fonction analytique à travers la potentialité réflexive qui s'y déploie? Et on sait combien les premières rencontres sont déterminantes quant aux modalités d'installation d'une cure et donc de son déroulement. La compréhension du matériel du patient repose sur le repérage et l'écoute du transfert du patient, mais aussi sur l'écoute par l'analyste de son propre fonctionnement mental.

L'analyste consultant peut se trouver devant le paradoxe de désirer affiner l'évaluation et de vouloir clore l'investigation assez rapidement. Alors que la problématique de l'interruption volontaire de transfert incitait à ne pas répéter les entretiens, aujourd'hui, lorsque le patient investit l'analyste consultant comme objet de transfert, il nous est apparu pertinent et nécessaire d'engager un travail élaboratif sur plusieurs entretiens. Car il importe alors de permettre un transfert du transfert, c'est-à-dire de donner au patient la possibilité d'investir le processus et la méthode, gage prédictif qu'il ne s'agira pas d'une cure interminable. L'interruption du processus de transfert pourrait être pensée comme épreuve de capacité au transfert, avec un premier travail de deuil du transfert sur le consultant. On s'écarte de «l'indication d'analyste» qui véhicule l'illusion d'un seul analyste possible, et questionne également la toute-puissance imagoïque du praticien faisant collusion avec la fantasmatisation du patient.

$\mathrm{Au}$ terme des rencontres consultatives, lorsque le processus de la rencontre s'est naturellement déployé, vient le temps de l'indication. Celle-ci s'accompagne d'une proposition de cadre: cure classique ou psychothérapie. En fonction de critères principalement cliniques, mais aussi sociaux et 
pécuniaires, le patient peut être inscrit sur une liste d'attente au CCTP où il pourra faire sa cure en gratuité. Il peut, s'il le demande, être adressé à un analyste dans le privé.

Lorsque les conditions de symbolisation et d'intelligibilité d'une cure analytique classique ou d'une psychothérapie ne sont pas réunies, l'analyste consultant propose d'autres cadres thérapeutiques psychanalytiques dont l'éventail est large et adapté aux différentes situations de la clinique: des psychodrames psychanalytiques individuels, des psychodrames collectifs de groupe ou en groupe, des traitements psychanalytiques de groupe, ou encore des entretiens thérapeutiques espacés pour des adultes jeunes ou en situations sociales de transition. Ces dernières approches ont un intérêt thérapeutique en elles-mêmes, mais elles peuvent aussi représenter un temps préliminaire à des traitements plus classiques. Ces différentes modalités thérapeutiques peuvent s'effectuer au CCTP, mais aussi relever d'orientations, soit vers d'autres établissements spécialisés, soit en pratique libérale pour les patients pour lesquels un engagement financier est possible et souhaitable, voire nécessaire parfois.

Lorsqu'une divergence trop importante apparaît entre une exigence de soins et la spécificité de la méthode analytique, les problématiques de la contre-indication, voire du refus, sont convoquées et doivent être reprises avec le patient. Il ne s'agit pas d'abandonner les patients mais de les accompagner vers des approches thérapeutiques mieux adaptées à leurs modes de fonctionnement et de les orienter vers d'autres structures: addictologie, prises en charge groupales, thérapies de soutien ou médicamenteuses.

Lorsqu'une prise en charge au centre est instaurée, le psychanalyste consultant peut être revu à tout moment du traitement, à la demande du patient ou du psychanalyste traitant. Dans le cas des traitements de groupe ou dans celui des entretiens espacés, le patient est engagé à revoir le consultant au moment de la fin de sa prise en charge.

Quel que soit le traitement proposé, il repose en tout point sur le respect de l'individu. Il requiert l'accord du patient et se fonde sur la notion de «consentement éclairé». Il importe donc que le consultant puisse expliciter à partir des données recueillies au cours des consultations ce qui motive sa proposition de traitement. Il est aussi très important de travailler avec le patient non seulement ses droits, mais également les contraintes pratiques qui pourraient entraver l'instauration du traitement: l'emploi du temps, les impératifs professionnels ou familiaux doivent s'avérer compatibles avec les horaires proposés. La prise en charge étant gratuite, la contrainte d'assiduité nécessite non seulement d'être énoncée, mais d'être élaborée avec le patient. 
La décision d'un traitement engage mutuellement thérapeute et patient. L'indication formulée par le consultant, proposée et acceptée par le patient, aboutit à l'inscription sur une liste d'attente. Le patient sera pris en traitement lorsqu'un analyste du Centre aura la disponibilité pour engager la prise en charge convenue avec la mise en place d'un cadre de travail avec des séances fixes. Le respect de ce cadre, de part et d'autre, fait partie intégrante de l'éthique du traitement. Il importe également que celui-ci puisse se mettre en place et commencer dans un délai raisonnable. Si le délai, compte tenu de la liste d'attente, paraît trop important, le consultant se mettra en relation avec d'autres institutions. Il peut aussi, en attendant le début du travail, proposer au patient des rencontres intermédiaires.

\section{L'écoute inter-analytique}

Le travail consultatif dans l'institution et la prise en charge d'un traitement psychanalytique en gratuité au CCTP reposent depuis une trentaine d'années (1989) sur l'investissement par les analystes de leur travail au Centre, le plaisir de l'échange inter-analytique et le libre choix d'y participer. Les analystes se réunissent deux fois par mois, il s'agit d'un groupe composé exclusivement d'analystes de la SPP travaillant au CCTP.

Avec la mise en place et la théorisation en après coup du double cadre ainsi que l'inscription des échanges inter-analytiques, J.-L. Donnet a ouvert un espace de travail et de tiercéisation qui peut être entendu comme possibilité d'une troisième règle fondamentale, à partir du modèle de la supervision avec la mise en situation de l'écoute en second. Avec cette différence notable qu'il n'y a pas d'enjeu institutionnel de validation même s'il reste toujours une dimension de reconnaissance par les pairs.

La question d'un indécidable concernant la théorie et la pratique, avec la nécessité de la suspension d'une théorie préalable, trouve son corollaire dans l'étude de la pratique et sa remise en cause constituant un jeu incessant d'allers et retours et d'après-coups féconds, telle qu'elle est mise en pratique au niveau du groupe clinique du CCTP.

Ce groupe se réunit deux fois par mois, le jeudi, et s'appelle depuis de nombreuses années le «groupe restreint» car n'y participent que les analystes qui travaillent au Centre. Après la mise en acte de la triangulation que représente la désunion consultation/traitement, le fonctionnement du groupe suit une méthodologie qui favorise une générativité associative et interprétative.

Ce sont les difficultés et les apories contre-transférentielles qui créent le désir ou la nécessité pour l'analyste de parler d'un patient, de s'interroger 
sur l'impasse clinique qu'il rencontre, de retrouver un positionnement de neutralité.

L'analyste qui souhaite parler de sa clinique présente brièvement quelques éléments de l'histoire du patient et de l'histoire de la cure, puis fait le récit de trois séances. Il est convenu qu'ensuite il garde le silence.

La discussion s'engage entre les participants devant l'analyste, qui n'intervient pas mais auquel la parole sera redonnée dans un troisième temps. Les variations des présentations sont autant d'éléments qui contribuent à la compréhension des mouvements transféro-contre-transférentiels à travers le respect ou non de la méthodologie dans le récit clinique, les faits sélectionnés, les évitements.

Il se met en place un travail associatif groupal autour d'un patient qui échappe à l'analyste dans un échange clinique soutenu par l'intérêt collectif pour le fonctionnement psychique et le travail analytique. On peut remarquer que les participants ont assez peu d'informations sur le patient, et ne reçoivent pas d'information complémentaire sur le cas présenté, ce qui convoque l'associativité groupale, à travers les identifications et contre identifications des participants au patient et à l'analyste. Le travail élaboratif du groupe se centre sur les éléments présentés et donne toute sa valeur à l'implication de l'analyste dans le matériel présenté. Cette écoute analytique groupale en second interroge la dynamique transféro-contre-transférentielle, les identifications aliénantes qui s'y déploient, soit lorsque l'interprétation ne permet pas de dégager le sens de traces mnésiques enfouies, soit lorsque les dénis et clivages entravent la construction d'un vide représentatif, tant au niveau du roman familial propre au patient que dans son histoire transgénérationnelle.

Dans un troisième temps, l'analyste reprend la parole, répond, ajoute des éléments cliniques, poursuit les élaborations proposées ou les discute, validant ou infirmant les hypothèses et les constructions proposées. En fin de séance, la reprise des consultations permet de retrouver la valeur prédictive de l'indication, sa pertinence ou ses insuffisances.

Au fil du temps, l'évaluation de l'incidence de la dualité consultation/ traitement a permis de renverser l'exigence institutionnelle en reconnaissance analytique d'un lien inter-analytique, tiercéisant et symbolisant.

Un colloque annuel ressaisit le travail du groupe restreint. Le thème du colloque se dégage au dernier moment, au cours du mois qui le précède, à partir d'une reprise des présentations de l'année. Ce colloque nous permet de partager notre travail avec un ensemble de collègues qui se trouvent alors en place de tiers. 
Dans cette époque où la valeur et l'efficacité du travail psychothérapique et psychanalytique sont contestées au prétexte d'un manque de critères objectifs, cette recherche nous permet d'apporter, à partir de notre pensée clinique, des éléments d'évaluation de notre pratique.

\section{Danielle Kaswin-Bonnefond} d.kaswin@wanadoo.fr

\section{Notes}

1. Sauf exception, tel le psychodrame exploratoire.

\section{Références}

Bolzinger, A. (2012), Portrait de Sigmund Freud: trésors d'une correspondance. Paris: Campagne première.

Donnet, J.-L. (2005). La situation analysante. Paris: Presses universitaires de France.

Donnet, J.-L. (1995). Le Divan bien tempéré. Paris: Presses universitaires de France.

Freud, S. (1998). Résultats, idées, problèmes. Tome II: 1921-1938. Paris: Presses universitaires de France.

Freud, S. (1938). Some Elementary Lessons in Psycho-analysis. The Standard Edition of the Complete Psychological Works of Sigmund Freud, t. XXIII. Paris: Presses universitaires de France, 1940.

Freud, S. (1910). À propos de la psychanalyse «sauvage». CEuvres complètes - Psychanalyse, t. X (p. 207-219). Paris: Presses universitaires de France, 1972.

Groddeck, G. (1977). Ça et moi. Lettres à Freud, Ferenczi et quelques autres. Paris: Gallimard. Stein, C. (2011). L'enfant imaginaire. Paris: Presses universitaires de France. 